between survivors and non-survivors $(3.20 \pm 1.34$ and $6.57 \pm$ 4.40 respectively, $p<0.001$ ).

Conclusion: In our study, APACHE III score and ED lengths of stay were higher versus other studies in Iran and other countries; which show more critical patients presenting to our hospital and limited ICU beds versus patients. This study results nevertheless there was significant difference between predicted and observed mortality rates, the APACHE III scoring system is applicable to evaluating care, treatment and prognosis of ED patients, as is used in ICU.

Prehosp Disaster Med 2011;26(Suppl. 1):s143-s144

doi:10.1017/S1049023X11004699

(P2-26) EMS-Assessed Mass Casualty Incident: A Pilot Surveillance

C.B. Park, ${ }^{1}$ S.D. Shin, ${ }^{2}$ G.J. Suh, ${ }^{2}$ J.O. Park, ${ }^{3}$ C.H. Kim ${ }^{4}$

1. Department of Emergency Medicine, Seoul, Korea

2. Emergency Medicine, Seoul, Korea

3. Jeju, Korea

4. Seoul, Korea

Background Recently the number of disaster and mass casualty incident (MCI) is increasing in Korea, but there have been few administrative reports or technical reports for disaster and MCI. An ad hoc basis data collection method is usually incomplete and outdated. This study was conducted to investigate the new surveillance system composed of EMS based real time survey and medical records based in-depth survey.

Methods: A retrospective review was conducted of the 119 fire department call center database and ambulance running sheets in one metropolitan city. The data on all transported patients with non-medical reasons (fire, rescue and others) between May 2006 and December 2008 was reviewed. We selected all data from the accidents which had more than 2 casualties to exam the feasibility and conducted in-depth surveillance based on medical records.

Results: The total number of accidents was 2,027 with 2,625 patients. The number of accidents which had more than 2 patients was 307 (total 898 patients) and more than 6 patients was 19 (total 176 patients). Among the "MCI" events, 15 cases were traffic accidents (125 patients, $71.0 \%), 4$ cases were fire (51 patients, 29\%). Total 142 medical records (80.7\%) were reviewed. Admission rate was $32.4 \%$ (46 patients) and overall mortality was 3.5\% (5 patients).

Conclusion: This nationwide public EMS system could contribute to the establishment of the systematic disaster database. Prehosp Disaster Med 2011;26(Suppl. 1):s144 doi:10.1017/S1049023X11004705

\section{(P2-27) Simulation of Transport During a Major} Incident

\section{B. Jezek, ${ }^{1}$ J. Vaněk, ${ }^{2}$ K. Antos, ${ }^{1}$ M. Prochazka ${ }^{2}$}

1. Faculty of Military Health Sciences, Hradec Kralove, Czech Republic

2. Department Of Public Health, Hradec Kralove, Czech Republic

Transportation capacities belong to the key factors of the response to a major incident. Available resources, both in terms of personnel and equipment, must be transported, usually by ambulances, to the incident location. In the other direction, casualties must be transported to hospitals and other health care facilities for further treatment. For this reason, the efficiency of the response is greatly determined by ambulance travel times and the ability of health care facilities to absorb large numbers of patients. We propose methods to compute the travel times to and from the incident location based on a classified road network. The methods take into account different attributes that depend on ambulance type and capacity, road quality, time of day, weather or actual traffic density. Correctly computed travel times are crucial not only for optimal deployment of all resources within the analyzed region, but also for the evaluation of the readiness of the emergency health care system for a major incident. We have included the methods in an agent-based simulation of transport during the response. From the simulation outputs and with the help of geographical information systems and information visualization methods we have synthesized maps that represent the capability of a region to absorb a major incident defined by a scenario. When combined with risk maps and maps of population density the synthesized maps allow emergency management authorities to find critical points and gaps in the emergency health care service.

Prehosp Disaster Med 2011;26(Suppl. 1):s144

doi:10.1017/S1049023X11004717

(P2-28) Collaboration Between Nurses and Physicians in the Emergency Department: An Indonesian Study

S. Suryanto

Faculty of Medicine, Malang, Indonesia

Background: Positive collaboration between nurses and physicians is essential in all areas of care especially in emergency practice. This is because it has a significant relationship with the quality, safety, accountability, and responsibility of care. Three areas are positively related to collaborative interaction between nurses and physicians: provider outcomes, patient outcomes, and organizational outcomes.

Aim: To examine nurses' and physicians' attitudes towards nurse-physician collaboration in the Emergency Department of Dr Saiful Anwar General Hospital, Malang, Indonesia.

Methods: The study was a comparative descriptive quantitative study using a modified Jefferson Scale of Attitude towards Physician-Nurse Collaboration. Data were collected from 47 nurses and 24 physicians who participated in the study. Descriptive statistics, parametric and non-parametric inferential statistics were used to determine group scores and to examine differences between groups, as well as to determine the relationship between demographic characteristics and participants attitudes.

Results: Emergency nurses had significantly more positive attitudes toward collaboration than emergency physicians $(p<0.001)$. Emergency nurses had significantly higher scores in three of four underlying factors of the instrument: "physician dominance", "nurse autonomy", and "caring as opposed to curing". The effects of gender, age, education, and experience in other hospitals on nurses' and physicians' attitude towards collaboration were not statistically significant. However, experience in the Emergency Department of Dr Saiful Anwar General Hospital was significantly related to participants' attitudes towards collaboration $(p=0.023)$.

Conclusions: The findings of this study indicate that both organizational and individual strategies should be developed to enhance the nurse-physician collaborative relationship. Interprofessional education may enhance health care professionals' 